\title{
Research at the IBM Zurich Laboratory
}

\author{
K. A. Müller, Zurich
}

(Senior Research Manager and Professor at Zurich University)

The Laboratory was established in 1956 as part of the IBM Research Division and since the spring of 1963 has been housed in its own location in Rüschlikon. It presently employs 124 people. Their activities reflect in close proportion the efforts of the whole Research Division which reports directly to the Corporate Management of the company. Approximately one third of the work is devoted to fundamental scientific research - briefly termed science - whereas the other two thirds of the available means are used for advanced technology projects. The latter may be defined as long-term applied research that encompasses comparatively high risks. In contrast, efforts which lead to specific products within a few years, and where the risk of failure is limited, are termed development projects. These are pursued within the various IBM product divisions. Quite a number of these development laboratories are located in Europe ; for instance at LaGaude in France, Hursley in England and Böblingen in the Federal Republic of Germany, to name but a few of the larger ones.

In Rüschlikon the main research in science is conducted in solid-state physics. It is largely investigatordependent, reflecting current interests of the scientific community, as well as the general hardware/technological interest of the company. Two advanced projects are pursued at the laboratory, one on Josephson technology and the other on computer sciences/communication technology which, due to their scope, are pursued as group efforts. As the physics and Josephson work is of more direct concern to physicists, the present article is restricted to these two topics. Particularly important results achieved in each are emphasized in order to put into perspective typical aspects of "science" and "advanced projects" in the IBM Research Division as carried out at the Zurich laboratory.

\section{Structural Phase Transitions (SPT)}

Research in solid state physics is centred around the study of phase transitions, surfaces and materials. In the following we shall describe that on structural phase transitions, SPT, a particular class of phase transitions, where significant advances have been achieved in the past decade. A fruitful cross-fertilization from the work, results, and ideas of the different research groups in our laboratory, as well as those of visiting scientists and predoctoral students has taken place, with physicists from about fifteen institutions and universities in France, Germany, Great Britain, Israel, Switzerland and the U.S. participating.

A phase transition is a cooperative phenomenon whereby the elementary interaction between atoms or molecules produces at sufficiently low temperatures, a specific ordering that shows up as a bulk property of the material. Ferromagnetism is the most familiar example. As microscopic interactions are disrupted by thermal vibrations, the ordering ceases above a characteristic temperature $T_{0} ;$ a phase transition occurs which depends upon the specific interactions of the material. Displacive SPT involve small displacements of atoms or tilts of groups of atoms in a crystal. They are seen in ferroelectric materials and have been widely studied in these materials because the onset of ferroelectric polarization provides a convenient property for measurement.

Displacive phase transitions also occur in strontium titanate, $\mathrm{SrTiO}_{3}$ which constitutes a more convenient model substance for exploring the phenomenon. It was discovered several years ago that below $T_{c}=110 \mathrm{~K}$, the quasi-rigid $\mathrm{TiO}_{6}$ octahedra start to rotate in an alternate manner around the cubic crystalographic direction as the temperature falls, see Fig. 1a. The rotation angle $\varphi$ the so-called order parameter, just like the magnetic or electric polarization in a ferromagnetic or ferroelectric material, is predicted by classical Landau theory to vary as
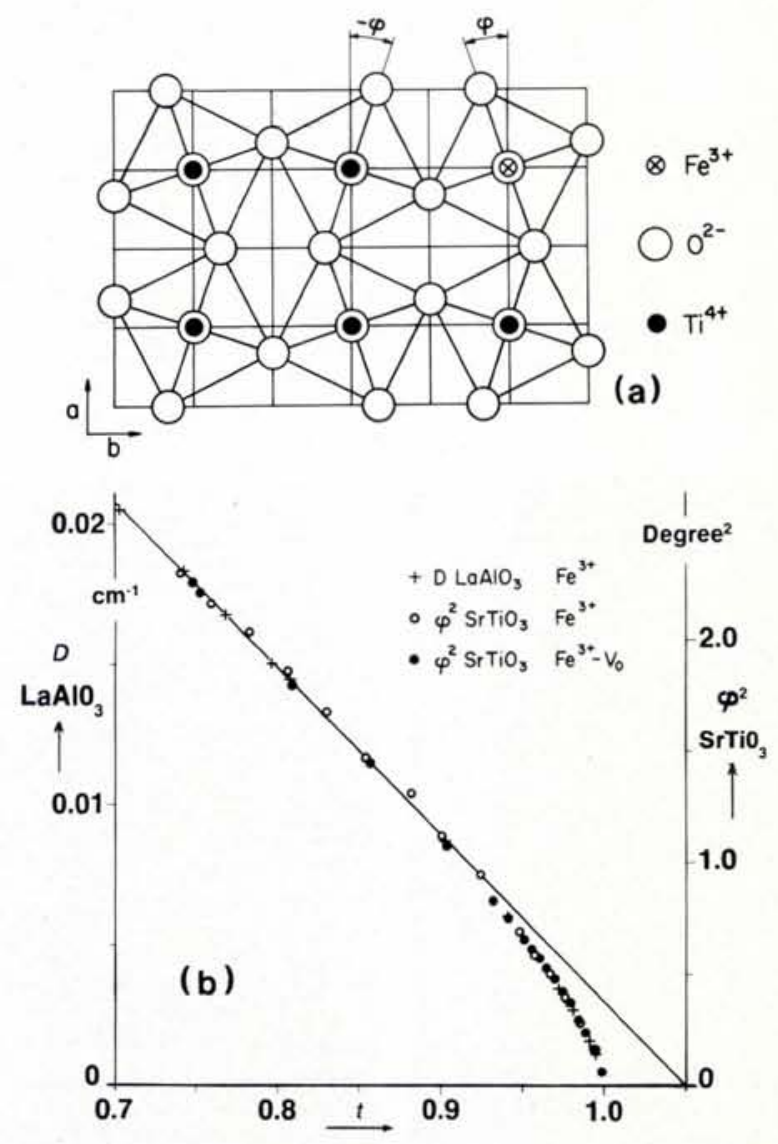

Fig. 1 (a) - Rotated oxygen octahedra around tetragonal c axis in $\mathrm{SrTiO}_{3}$ below $\mathrm{T}_{\mathrm{c}}$ $=110 \mathrm{~K}$. The presence of an $\mathrm{Fe}^{3+}$ substitutional point defect for the paramagnetic resonance experiment is indicated. (b) $\varphi^{2}$ in $\mathrm{SrTiO}_{3}$ and $D \propto \phi^{2}$ in $\mathrm{LaAlO}_{3}$ versus $t$ $=\left(T_{c}-T\right) / T_{c}$ between 0.7 and 1, showing the crossover from Landau to critical behaviour. 
$\varphi \propto\left(T_{c}-T\right)^{1 / 2}$. But whereas such a temperature dependence has been observed in ferroelectric transitions, an electron paramagnetic resonance experiment that allowed $\varphi(T)$ to be determined with high precision, showed that in $\mathrm{SrTiO}_{3}$ and $\mathrm{LaAlO}_{3}$, near $T_{c}$ $\varphi \propto\left(T_{c}-T\right)^{0.3}$, although at lower temperatures the square root relationship applies (Fig. 1b). The explanation is that classical theory neglects correlated clusters of ordered atoms whose size grows as $T_{c}$ is approached. The details of how such clusters form, grow and decay near $T_{c}$ are central to an understanding of phase transitions.

A recent development which laid the basis for a very general theory of phase transitions, called renormalization-group (RG), was initiated by Kenneth Wilson and Michael Fisher of Cornell University. This theory describes the wide class of transitions in which the order parameter is continuous at $T_{c}$, be they ferromagnetic, structural or of other types. What counts is the symmetry, the number of components of the order parameter, and the dimensionality of the lattice. Breaking the symmetry by an external force such as uniaxial stress must lead to a different critical behaviour. Thus measurments on $\mathrm{SrTiO}_{3}$ stressed along a [111] direction recently verified $R G$ predictions for the so-called three-state Potts model. The latter is a nontrivial generalization of the wellknown two-state Ising model, and is applicable to transitions where the order parameter can assume three distinct, but equivalent values or orientations, i.e., can be reoriented but not reversed. Another success was achieved with $\mathrm{RbCaF}_{3}$ which undergoes the same type of SPT as $\mathrm{SrTiO}_{3}$, although it is first order, due to strongly anisotropic fluctuations of cubic symmetry. As predicted by the $R G$, uniaxial stress nearly along a cubic [100] direction restored a continuous transition at a so-called "tricritical point". In its vicinity, a different dependence was observed, in agreement with RG theory namely $\varphi \propto\left(T_{c}-T\right)^{1 / 6}$.

In a displacive SPT, on approaching $T$ from above, a vibrational mode becomes soft ; its frequency $\omega_{s}$ tends to vanish, while the corresponding displacement pattern "freezes-in" to produce the distorted lattice corresponding to the low temperature phase. Since 1971, neutron and light scattering experiments have indicated that the soft mode does not actually freeze completely in $\mathrm{SrTiO}_{3}$, as predicted by classical theory, i.e., $\omega_{s}\left(T=T_{c}\right) \neq 0$, and that a "central peak" centred at $\omega=0$ appears in the correlation spectrum of fluctuations of $\varphi$. It was recognized that this phe-

Fig. 2 (Left) - Photomicrograph of a portion of the Josepson memory model showing sixteen memory cells. Each cell contains two Josephson junctions. A bit of information is stored as the presence or absence of a current circulating through the junctions. The current persists indefinitely unless the junctions are switched into a resistive state. (Right) - A portion of the control circuitry for a Josephson memory chip. The Josephson junctions are the lozenge-shaped areas. This shape produces better switching characteristics in this application than the more usual rectangular shape.
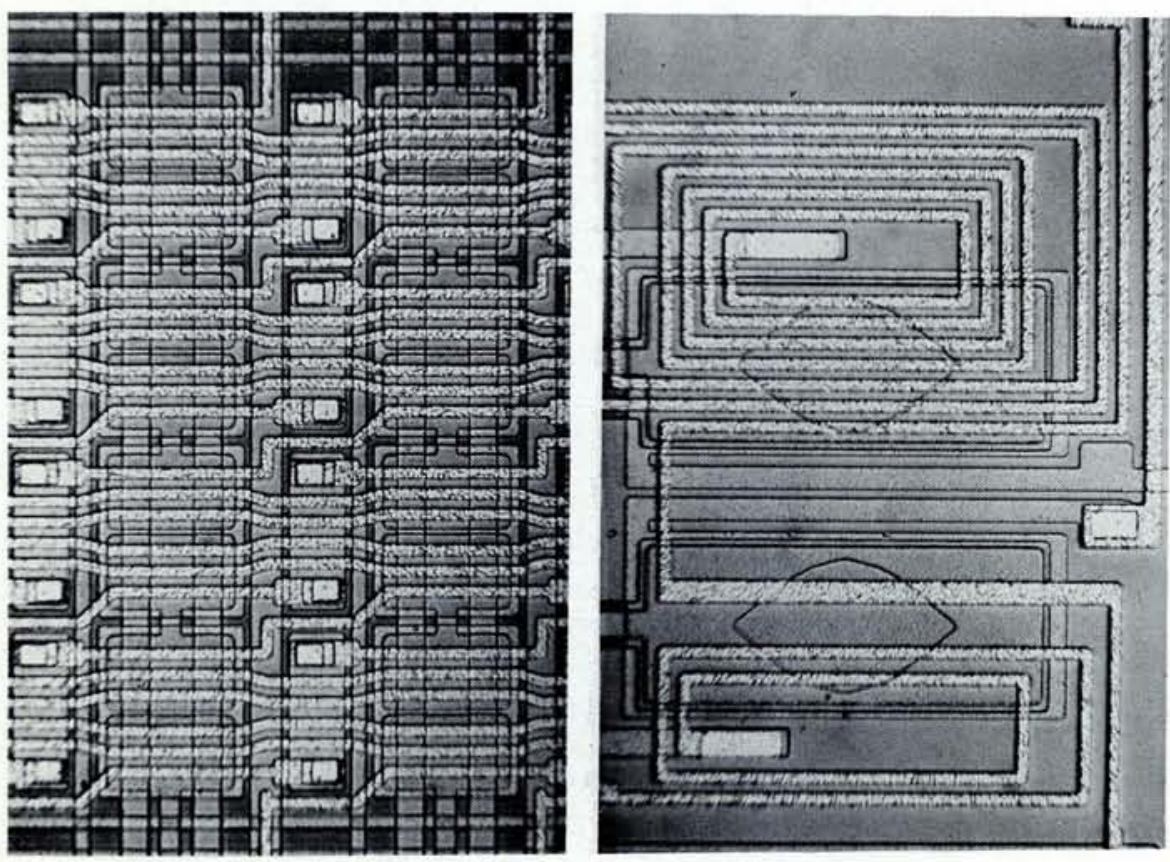

nomenon results from highly nonlinear interactions of the atoms which could not be analysed by either conventional or self-consistent perturbation theory treatments of anharmonic lattice dynamics. At the laboratory a novel molecular dynamics technique was developed to simulate numerically, using large scale computations, ensembles of displacive and related systems exchanging energy with a heat reservoir at a constant temperature. Simulations for a two-dimensional lattice showed for the first time that the central peak is due to the decay and motion of correlated clusters and, in particular, that the walls between clusters propagate as localized excitations reminiscent of the so-called "solitons" found in many branches of physics and applied mathematics. These simulations also indicated that, on approaching $T_{c}$, a displacive transition resembles more and more an order-disorder one, in which particular atoms move in doublewell potentials, as in the ferroelectric $\mathrm{KH}_{2} \mathrm{PO}_{4}$. Quite recently this crossover from displacive to order-disorder behaviour was shown through a paramagnetic-resonance experiment to account quantitatively for the nonvanishing soft mode in $\mathrm{SrTiO}_{3}$.

In order-disorder systems, the critical dynamics of the crystal is of a relaxational rather than soft-mode character, i.e., all fluctuations occur near $\omega=0$. If impurity effects are absent, no further dynamics should exist, i.e., no additional "central peak" is expected. This was also demonstrated recently in Rüschlikon by Brillouin scattering on annealed $\mathrm{KH}_{2} \mathrm{PO}_{4}$ crystals. If, however, slowly relaxing impurities are present, like $\mathrm{Cr}^{5+}$ in $\mathrm{KH}_{2} \mathrm{PO}_{4}$, then an additional central peak appears. It was expected that the correlation length characterizing the extent of an average cluster should diverge at $T_{c}$ but this need not always be the case. Again in $\mathrm{KH}_{2} \mathrm{PO}_{4}$, in the presence of an electric field, a continuous transition without microscopic critical fluctuations was found, so the system behaves exactly as Lev Landau proposed in 1937.

\section{Josephson Technology}

IBM Research has an ambitious programme to develop an entirely new computer technology based on Josephson tunnel junctions. These devices, which depend on superconductivity and thus operate only at low temperatures, combine high switching speed with very low heat generation. 
This potentially permits them to outperform transistor circuits. To build a computer out of Josephson devices, however, requires development of a whole new technology - one that can borrow only some portions of today's semiconductor technology. Thus research efforts include materials evaluation, design of device and circuit structures, development of processing techniques, and packaging designs. The work at the Zurich laboratory concentrates on memory and storage technology. A parallel effort at the IBM Watson Research Center in Yorktown, New York, is devoted to logic circuitry and packaging.

A Josephson junction consists of a layer of oxide only tens of atom diameters thick separating two films of superconducting metal. Electric current can pass through the insulating layer in two ways. If the current is small and no magnetic field is present, the insulating layer acts as a superconductor itself : there is no voltage drop across it. If the current exceeds a critical value, or if a magnetic field greater than a critical intensity is present, a voltage drop appears across the junction. The oxide layer in this state conducts current by incoherent tunnelling of unpaired electrons. These are the two states that permit Josephson junctions to serve as computer switches.

Another aspect of superconductivity permits such switches to form the basis of memory devices. A basic property of superconducting metals is that, below a certain critical magnetic field, they exclude magnetic flux they are perfectly diamagnetic. A memory device based on this principle uses one or more Josephson junctions in a superconducting loop. When a circulating current is established in the loop, the magnetic field generated inside it remains trapped as long as the metal films forming the loop remain superconducting. The circulating current persists once established, and its presence or absence can represent a bit of information. When the Josephson junction is switched into the voltage (non-superconducting) state by a small external magnetic field, the magnetic field in the loop collapses as the loop is no longer superconducting. This collapse induces a voltage pulse in the circuit indicating that a current had been stored in the cell. If the cell had no circulating current, switching it to the voltage state would produce no change in magnetic flux and thus no voltage pulse. This is one way of reading stored information. The external field needed to cause this switching action

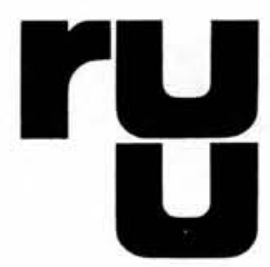

Fields of Activity

\section{Information}

Proposals

A vacancy exists in the Faculty of Mathematics and Natural Science of the State University of Utrecht, The Netherlands, for a

\title{
Professor of Technical Physics
}

\begin{abstract}
The successful candidate will be expected to carry out research in the area of material science within the technical physics department and to collaborate with the departments of solid state physics and atomic and molecular physics. Further, contacts with departments and research institutes outside the faculty will be encouraged. The development of the current research programme of the department in the field of energy will be decided after consultation with the appointee. Industrial or other relevant experience is desirable. The appointee will participate in the general physics teaching programme as well as in more specialized education in technical physics.
\end{abstract}

Further information and details of the present structure of the department can be obtained from the chairman of the selection committee :

Professor H.W. de Wijn, Fysisch Laboratorium der Rijksuniversiteit,

P.O. Box $80.000,3508$ TA Utrecht, The Netherlands, Tel. (030) - 532414.

Written applications or suggestions for suitable candidates should be submitted within four weeks of the appearance of this advertisement to the chairman of the selection committee.

is produced by a current in a control line on top of the Josephson junction.

A remarkable property of this kind of memory cell is that it can store a single quantum of magnetic flux, $2.07 \times 10^{-15} \mathrm{~Wb}$ - the ultimate economy in magnetic memory. The amount of energy stored in such a memory cell can be less than $10^{-18}$ joule. Zurich laboratory scientists were among the first to propose single flux quantum loops as Josephson memory devices and have concentrated on their development as a candidate for main memory in a Josephson computer. The basic cell consists of two Josephson junctions and an inductance and this circuit has turned out to be the one most suitable for single flux quantum storage.

Single flux quantum cells have been fabricated and found to operate as predicted by theoretical models. More recently, a model for a 16,000-bit memory chip was fabricated and tested. Experiments showed that substantial arrays of cells can be fabricated to acceptable tolerances in the laboratory, that the decoding, driving and sensing circuits are satisfactory, and that the switching of cells does not excessively disturb the state of other cells. These results indicate that large capacity single flux quantum Josephson memories should be feasible.

Most work on Josephson switching devices to date has used alloys of lead for the superconducting lines. While using these alloys for exploratory memory arrays, researchers in Rüschlikon have at the same time been exploring alternative technologies, based, for example, on the use of niobium for the electrodes. Niobium is a good superconductor and has the advantage over lead alloys of having greater stability in cycling between room temperature and liquid helium temperature. It also presents some problems. Niobium has several oxidation states, which are not well understood, and its oxides have a high dielectric constant. This increases the capacitance of a Josephson junction and thus reduces its switching speed. The speed may be acceptable for main memory, however. Ultimately, commercial considerations will decide as to whether such devices will be used in future computers.

Parts of this article have been adapted from a more detailed brochure on the Zurich Research Laboratory which is available on request. 